\title{
Infliximab treatment in ankylosing spondylitis: an observational study
}

\author{
S N Nikas, Y Alamanos, P V Voulgari, X I Pliakou, C G Papadopoulos, A A Drosos
}

Ann Rheum Dis 2005;64:940-942. doi: 10.1136/ard.2004.029900

\begin{abstract}
Objective: To investigate efficacy, toxicity, and drug discontinuation in patients with ankylosing spondylitis (AS) treated with infliximab.

Methods: 35 patients with AS with mean (SD) age 42.5 (12.6) years and mean (SD) disease duration 14.5 (8.0) years were studied for 2 years. Patients entering the study had a negative tuberculin skin test, were fully informed about the treatment, and were followed up regularly. Infliximab, $5 \mathrm{mg} / \mathrm{kg}$ weight, was given intravenously at weeks $0,2,6$, and every 8 weeks thereafter. Data concerning infliximab tolerability, adverse events, interval, and drug discontinuation were all recorded. Clinical improvement according to the BASDAI and the Ankylosing Spondylitis Assessment Study group (ASAS) 20\%, 40\%, and ASAS 5/6 response criteria were recorded.

Results: After 1 year, 20 (57\%) patients achieved the BASDAI 50\% response criteria, $25(71 \%)$ achieved ASAS $20 \%, 23(66 \%)$ reached ASAS $40 \%$, and $18(51 \%)$ attained ASAS 5/6. After 2 years' treatment, 11 (31\%) patients achieved BASDAI 50\% response criteria, $14(40 \%)$ ASAS $20 \%, 11$ (31\%) ASAS 40\%, and 9 (26\%) ASAS 5/6. Clinical improvement was associated with an improved BASFI and reduction of CRP. After 2 years' treatment, "infliximab survival" was $89 \%$. Treatment was well tolerated and adverse events were mild; 3 patients discontinued the study. Conclusion: Infliximab was effective, safe, and well tolerated in patients with AS.
\end{abstract}

A nkylosing spondylitis (AS) is a chronic inflammatory disease affecting primarily the axial skeleton, leading to functional disability and premature death. ${ }^{1}$ An extensive rationale exists for the use of tumour necrosis factor $\alpha$ inhibitors (including infliximab) in AS, and a growing body of evidence has shown the clinical efficacy of this approach. ${ }^{2}$ The efficacy and safety of infliximab have been established in a number of short, open label, randomised controlled trials in patients with $\mathrm{AS}^{3-7}$ As in rheumatoid arthritis (RA), treatment of AS must be continued because disease activity returns a few weeks after infliximab is stopped. On the other hand, serious adverse events, including predisposition to bacterial infections, reactivation of tuberculosis, and others, are some of the disadvantages of infliximab treatment.

Thus, rheumatologists and physicians should know (a) for how long infliximab is effective and safe; and $(b)$ which are the most common and hazardous adverse events. To examine the above questions, we investigated infliximab efficacy, drug survival, and reasons for drug discontinuation during the disease course in an observational study of patients with refractory AS.

\section{MATERIALS AND METHODS}

From June 2001 until December 2003, 41 patients with AS were recruited. All patients fulfilled the modified New York criteria for $\mathrm{AS}^{8}{ }^{8}$ Patients were fully informed about the treatment regimen and were followed up at predefined times according to a standardised protocol. Infliximab was given intravenously (infusion time $>2$ hours) in a loading dose of $5 \mathrm{mg} / \mathrm{kg}$ weight at weeks $0,2,6$, and every 8 weeks thereafter. If the therapeutic response was insufficient, then the interval was shortened to 6 or to 4 weeks. The exclusion criteria have been reported elsewhere.

Data concerning infliximab efficacy, tolerability, concomitant treatment, adverse events, and discontinuation were all recorded. Data on clinical improvement according to the Bath Ankylosing Spondylitis Disease Activity Index (BASDAI), and the Ankylosing Spondylitis Assessment Study Group (ASAS) $20 \%$ and $40 \%$ and ASAS 5/6 response criteria, ${ }^{10}$ were also recorded. All patients had their last follow up by June 2004.

\section{Definitions}

- Active disease was defined if patients had BASDAI $\geqslant 40 / 100^{9}$ and $\mathrm{C}$ reactive protein $(\mathrm{CRP}) \geqslant 10 \mathrm{mg} / \mathrm{l}$ (normal value $<6 \mathrm{mg} / \mathrm{l})$.

- Refractory disease was defined by the failure of at least two non-steroidal anti-inflammatory drugs (NSAIDs) during a single 3 month period, failure of intra-articular steroids if indicated, and failure of sulfasalazine in patients with peripheral arthritis.

- A response to treatment according to ASAS criteria requires improvement of at least $20 \%$ and absolute improvement of at least 10 units on a scale of $0-100$ in three of the following four domains: (a) patient's global assessment of the disease activity; (b) pain; (c) function (in this study the Bath Ankylosing Spondylitis Functional Index (BASFI) score) ${ }^{11}$; and $(d)$ inflammation (in this study the mean duration of morning stiffness related to the BASDAI, and $100 \mathrm{~mm}$ visual analogue scale (VAS) scores) and the absence of deterioration by $20 \%$ and by $10 \%$ of units in the four domains. ${ }^{12}$

- Lack of efficacy was defined as patients not fulfilling the BASDAI $20 \%$, as well as the ASAS $20 \%$ response criteria.

- Failure of drug treatment was defined as patients who stopped receiving the drug for more than 2 months because of lack of efficacy.

- Adverse drug reactions were defined as patients who had reactions that required permanent discontinuation of

Abbreviations: AS, ankylosing spondylitis; ASAS, Ankylosing Spondylitis Assessment Study Group; BASDAI, Bath Ankylosing Spondylitis Disease Activity Index; BASFI, Bath Ankylosing Spondylitis Functional Index; CRP, $C$ reactive protein; HACA, human antichimeric antibodies; NSAIDs, non-steroidal anti-inflammatory drugs; RA, rheumatoid arthritis; VAS, visual analogue scale 
Table 1 Clinical and laboratory data of patients with AS treated with infliximab

\begin{tabular}{ll}
\hline Patients (n) & 35 \\
Male/female & $34 / 1$ \\
Age (years), mean (SD) & $42.5(12.6)$ \\
Disease duration (years), mean (SD) & $14.5(8.0)$ \\
Peripheral arthritis, No (\%) & $2(6)$ \\
History of uveitis, No (\%) & $11(31)$ \\
Methotrexate intake, No (\%) & $3(9)$ \\
Sulfasalazine intake, No (\%) & $3(9)$ \\
Prednisone intake (5 mg/day), No (\%) & $4(11)$ \\
NSAIDs, No (\%) & $35(100)$ \\
HLA-B27, No (\%) & $31(89)$ \\
BASDAl, mean (SD) & $57.2(18.5)$ \\
CRP (mg/l), mean (SD) & $25.6(17.0)$ \\
\end{tabular}

infliximab owing to life threatening conditions, or because of intolerability.

- Discontinuation was decided when drug treatment failed or patients had adverse drug reactions.

\section{Monitoring}

A complete blood count with differential and platelet count, as well as serum values of liver enzymes, bilirubin, albumin, glucose, creatinine, and urine analysis were obtained before treatment and at each patient's visit. Finally, blood serum from patients was stored for the measurement of antibody profile.

\section{Statistical analysis}

Standard methods of survival analysis (Kaplan-Meier) were used, in which infliximab termination due to side effects and/ or lack of efficacy, and/or failure of drug treatment were taken as the end points.

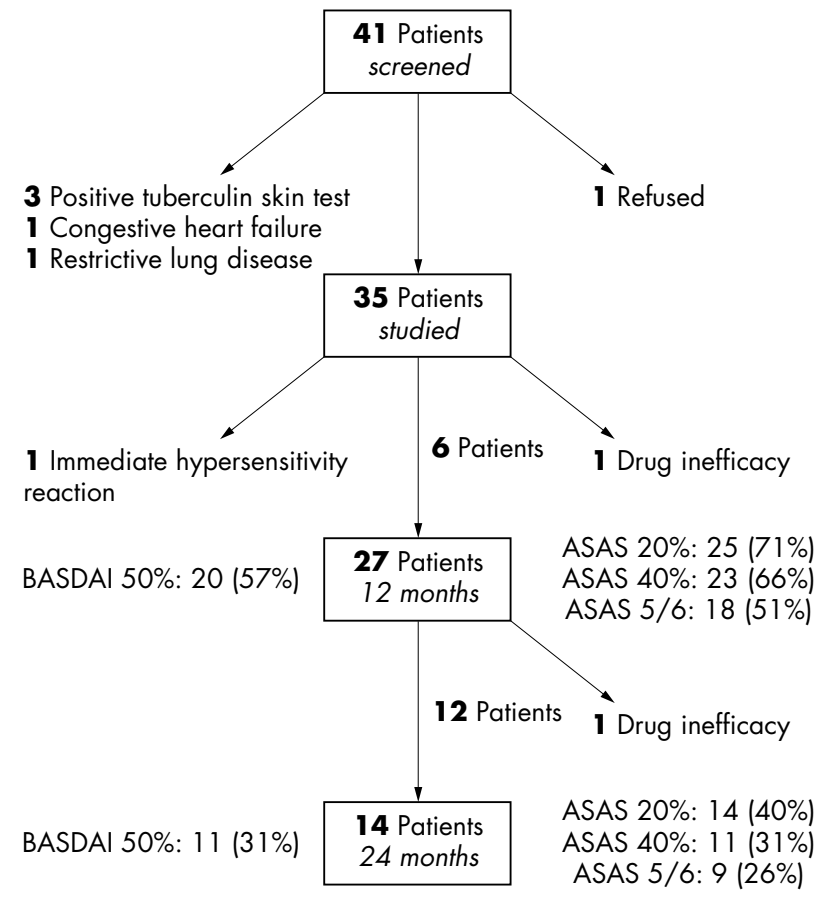

Figure 1 Study profile and response to treatment. The lateral arrows represent the patients who did not meet entry criteria or discontinued the study. The middle arrows represent the patients who continued infliximab treatment but were not followed up for a full 1 or 2 years. Percentages of response are calculated on the basis of 35 patients presented at entry.

\section{RESULTS}

During the recruitment period 41 patients with AS were enrolled. Of these, one refused treatment and five were excluded from the study. Thus, 35 patients were investigated. Table 1 shows the demographic, clinical, and laboratory data of patients at entry.

All patients had axial disease, two patients presented with peripheral arthritis and 11 had a history of anterior uveitis. All patients had active disease as evaluated by the high BASDAI score and high levels of CRP (table 1). The mean (SD) duration of morning stiffness related to BASDAI was 60.9 (27.7) minutes and that related to the VAS was 65.0 (28.0) minutes. Figure 1 shows the study profile and the response to treatment according to the BASDAI and ASAS criteria. After 2 years of infliximab treatment a significant number of patients achieved the BASDAI and ASAS response criteria.

In addition, infliximab treatment resulted in a rapid and significant improvement in the BASDAI and BASFI scores and a durable response for 24 months. This clinical and functional improvement was associated with the reduction of acute phase reactants as measured by CRP levels (fig 2). No correlation of BASDAI and CRP was found at baseline and after treatment. Furthermore, no significant improvement in spinal mobility, as measured by the Schober test, was noted. After the first year of treatment, the "survival rate" of infliximab was $94 \%$, while this rate was $89 \%$ after the second year. The treatment was well tolerated with mild adverse events.

Eleven patients (31\%) experienced adverse events, mainly infections and allergic drug reactions. All these adverse events, except one, resolved without sequelae. One patient withdrew from the study due to an immediate hypersensitivity reaction after the third infusion, and two more patients had to stop because of drug inefficacy. The first was a man with a longstanding history of axial disease and hip involvement, who discontinued the study after the sixth infliximab infusion. The second was also a man with axial disease, and severe scoliosis and kyphosis, who discontinued the study after the ninth infusion. These two patients had had no response to treatment since the beginning, thus the interval between infusions had been shortened from 8 to 6 weeks. The first patient's interval had been shortened after the fourth infusion, and the second patient's interval after the fifth. No further improvement was achieved. The interval between infusions was shortened from 8 to 6 weeks for four further patients. These patients initially responded well to infliximab treatment, but 10 days before the next infusion they experienced neck pain and stiffness. In one patient the interval was shortened after the eighth infusion, and in three patients after the tenth infusion. All responded well to this treatment strategy.

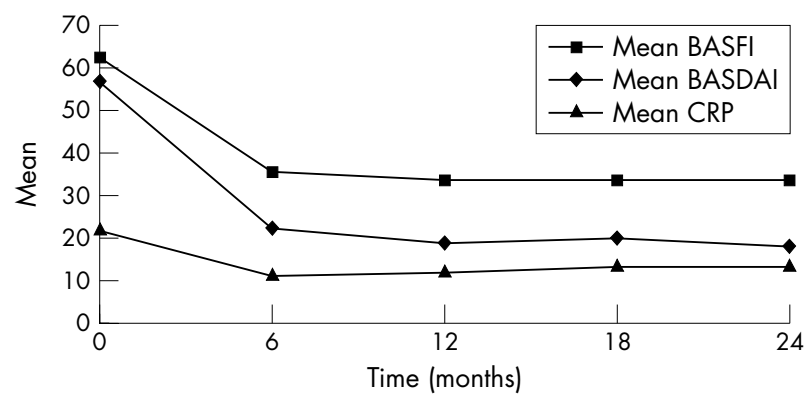

Figure 2 Improvement of the BASDAI and BASFI and reduction of CRP. 
Finally, eight (23\%) patients developed positive antinuclear antibodies, with a fine speckled pattern, in titres ranging between $1 / 160$ and 1/640. No other antibodies were detected.

\section{DISCUSSION}

AS is a chronic inflammatory rheumatic disease affecting primarily young men, with an estimated prevalence of $0.15-$ $0.8 \%{ }^{13}$ Tumour necrosis factor $\alpha$ inhibitors have been shown to be highly efficacious in patients with active AS. Several placebo controlled and open trials have shown that active AS responds dramatically to infliximab. ${ }^{3-7}$ As in RA, the treatment of AS must be continued because the disease activity returns a few weeks after infliximab is stopped. On the other hand, serious adverse events may occur during infliximab treatment. One main finding of the present study during the observation period of 2 years is that infliximab was effective as measured by the BASDAI $50 \%$, the ASAS $20 \%$ and $40 \%$, and ASAS 5/6 response criteria. The second finding of our results was the significant functional improvement as evaluated by the BASFI score, which was associated with the reduction of acute phase reactants. The third point is that $89 \%$ of our patients continued to have beneficial effects after the second year of treatment.

The efficacy and safety of infliximab and its survival in the present study of patients with AS is greater than expected. The absence of serious adverse events is probably attributed to the strict inclusion criteria used. The drug survival for infliximab treatment in AS has not been reported so far. Loss of infliximab survival may be explained by the generation of human antichimeric antibodies (HACA). These antibodies appeared to be associated with lower serum infliximab concentrations, and may be related to a shorter duration of response after repeated infliximab doses, as reported for patients with RA. Concomitant administration of methotrexate appears to reduce the formation of HACA. However, in our study only three patients were taking methotrexate.

Another point to take into consideration is the higher infliximab dosage used, as compared with patients with RA. Formation of HACA may be inversely related to the infliximab dose. HACA were found in 53, 21, and $7 \%$ of patients with RA receiving infliximab 1,3 , or $10 \mathrm{mg} / \mathrm{kg}$, respectively, 12 weeks after the last of five infusions of infliximab. ${ }^{14}$ It has been suggested that higher doses of infliximab may be associated with immunological tolerance. ${ }^{14}$ This may explain the high "survival rate" of infliximab in our patients with AS and its beneficial effects for a period of 2 years. Limited data are available about the optimal dosage of infliximab in spondyloarthropathies. In a small study it was shown that a dose of $5 \mathrm{mg} / \mathrm{kg}$ was better than $3 \mathrm{mg} / \mathrm{kg} \cdot{ }^{15}$ However, the lower dose of infliximab seems to work as well.

The results of this observational study suggest that infliximab treatment was effective, safe, and well tolerated in patients with AS. The survival of infliximab after 2 years of treatment was $89 \%$. However, further long term, controlled and observational studies, with large numbers of patients, are needed to validate our results.

\section{Authors' affiliations}

S N Nikas, P V Voulgari, X I Pliakou, C G Papadopoulos, A A Drosos, Rheumatology Clinic, Department of Internal Medicine, Medical School, University of loannina, loannina, Greece

Y Alamanos, Department of Hygiene and Epidemiology, Medical School, University of loannina, loannina, Greece

Correspondence to: Professor A A Drosos, Rheumatology Clinic, Department of Internal Medicine, Medical School, University of loannina, 45110 loannina, Greece; adrosos@cc.voi.gr

Accepted 21 November 2004

Published Online First 25 November 2004

\section{REFERENCES}

1 Lehtinen K. Mortality and causes of death in 398 patients admitted to hospital with ankylosing spondylitis. Ann Rheum Dis 1993;52:174-6.

2 Braun J, Bollow M, Neure L, Seipelt E, Seyrekbasan F, Herbst H, et al. Use of immunohistologic and in situ hybridization techniques in the examination of sarcoiliac joint biopsy speciments from patients with ankylosing spondylitis. Arthritis Rheum 1995;38:499-505.

3 Brandt J, Haibel H, Cornely D, Golder W, Gonzalez J, Reddig J, et al. Successful treatment of active ankylosing spondylitis with the anti-tumor necrosis factor alpha monoclonal antibody infliximab. Arthritis Rheum 2000;43:1346-52.

4 Van Den Bosch F, Kruithof E, Baeten D, Herssens A, de Keyser F, Mielants H, et al. Randomized double-blind comparison of chimeric monoclonal antibody to tumor necrosis factor alpha (infliximab) versus placebo in active spondylarthopathy. Arthritis Rheum 2002;46:755-65.

5 Braun J, Brandt J, Listing J, Zink A, Alten R, Golder W, et al. Treatment of active ankylosing spondylitis with infliximab: a randomized controlled multicentre trial. Lancet 2002;359:1 187-93.

6 Braun J, Brandt J, Listing J, Zink A, Alten R, Burmester G, et al. Long term efficacy and safety of infliximab in the treatment of ankylosing spondylitis: an open, observational, extension study of a three-month, randomized, placebocontrolled trial. Arthritis Rheum 2003;48:2224-33.

7 Temekonidis TI, Alamanos Y, Nikas SN, Bougias DV, Georgiadis AN, Voulgari PV, et al. Infliximab therapy in patients with ankylosing spondylitis: an open label 12 month study. Ann Rheum Dis 2003;62:1218-20.

8 van der Linden S, van Valkenburg HA, Cats A. Evaluation of diagnostic criteria for ankylosing spondylitis. A proposal for modification of the New York criteria. Arthritis Rheum 1984;27:361-8.

9 Garrett S, Jenkinson T, Kennedy LG, Whitelock H, Gaisford P, Calin A. A new approach to defining disease status in ankylosing spondylitis: the Bath Ankylosing Spondylitis Disease Activity Index. J Rheumatol 1994;21:2286-91.

10 Brandt J, Listing J, Sieper J, Rudwaleit M, van der Heijde D, Braun J. Development and preselection of criteria for short-term improvement after anti-TNF $\alpha$ therapy in ankylosing spondylitis. Ann Rheum Dis. 2004;63: 143844 doi, 10.1136/ard.2003.016717 [published Online First:25 March 2004].

11 Calin A, Garrett S, Whitelock H, Kennedy LG, O'Hea J, Mallorie P, et al. A new approach to defining functional ability in ankylosing spondylitis: the development of the Bath Ankylosing Spondylitis Functional Index. J Rheumatol 1994;21:2281-5.

12 Anderson JJ, Baron G, van der Heijde D, Felson DT, Dougados M. Ankylosing spondylitis assessment group preliminary definition of short-term improvement in ankylosing spondylitis. Arthritis Rheum 2001;44:1876-86.

13 Alamanos Y, Papadopoulos NG, Voulgari PV, Karakatsanis A, Siozos C, Drosos AA. Epidemiology of ankylosing spondylitis in Northwest Greece, 1983-2002. Rheumatology (oxford) 2004;43:615-18.

14 Maini RN, Breedveld FC, Kalden JR, Smolen JS, Davis D, Macfarlane JD, et al. Therapeutic efficacy of multiple intravenous infusions of anti-tumour necrosis factor alpha monoclonal antibody combined with low-dose weekly methotrexate in rheumatoid arthritis. Arthritis Rheum 1998;41:1552-63.

15 Brandt J, Haibel H, Reddig J, Sieper J, Braun J. Successful short term treatment of severe undifferentiated spondyloarthropathy with the anti-tumor necrosis factor-alpha monoclonal antibody infliximab. J Rheumatol 2002;29:1 18-22. 